\title{
The Importance of Prehabilitation in Liver Transplantation
}

\author{
Aaron M. Williams ${ }^{1} \cdot$ Seth Waits $^{1} \cdot$ Michael J. Englesbe ${ }^{1}$
}

Published online: 10 October 2015

(C) Springer International Publishing AG 2015

\begin{abstract}
Over 15,000 patients are listed for liver transplantation across the USA while only 6500 transplants are performed each year. Given the realities of this profound organ shortage, optimal patient preparation is important to assure good outcomes. In recent years, frailty and sarcopenia have emerged as important predictors of post-transplant mortality. Potentially, these risk factors may be remediable with preoperative preparation. Efforts to improve disease management and physical conditioning could not only optimize patients for liver transplantation but could also improve outcomes among those who do not undergo transplantation.
\end{abstract}

Keywords Frailty $\cdot$ Sarcopenia $\cdot$ Prehabilitation · Preoperative optimization $\cdot$ Liver transplantation

\section{Introduction}

More than 15,000 patients across USA are currently on the waiting list for liver transplantation [1]. Despite a growing need, only 6500 liver transplants are performed each year. Given the realities of this profound organ shortage, optimizing graft and recipient survival has become increasingly important.

The model for end-stage liver disease (MELD)-based allocation system has decreased waitlist mortality, but at the time

This article is part of the Topical Collection on Liver Transplantation

Michael J. Englesbe

englesbe@umich.edu

1 Department of Surgery, Division of Transplantation, University of Michigan Health System, 1500 East Medical Center Drive, 2926A Taubman Health Center, Ann Arbor, MI 48109-5300, USA of transplant, most patients are gravely ill [2]. Despite this severity of illness at the time of transplantation, it is an expectation to maintain excellent recipient survival. These pressures have focused attention on recipient readiness for surgery, a potentially modifiable risk factor for patient survival.

Within this context, frailty and sarcopenia are concepts that have gained momentum in understanding the clinical trajectory in patients with end-stage liver disease (ESLD) [3-7]. Frailty and sarcopenia are not only important to clinical outcomes for patients with ESLD but may be modifiable during the waiting period for transplantation [8]. With this paper, we will investigate potentially modifiable risk factors for patients before, during, and after liver transplantation. We will also consider how preoperative preparation - termed prehabilitationmay improve outcomes in this high-risk population.

\section{Frailty}

Frailty is defined as the biologic syndrome of decreased reserve and resistance to stressors, resulting from cumulative declines across multiple physiologic systems and causing vulnerability to adverse outcomes [9]. Seen particularly in elderly patients, it is characterized by low functional reserve, easy tiring, mood disturbance, accelerated osteoporosis, decreased muscle strength, and high susceptibility to disease. The pathophysiology of frailty remains elusive, but it is thought to be secondary to an aggregate decline in numerous domains including the neurovascular, skeletal, inflammatory, and endocrine systems. For patients with cirrhosis, liver failure accelerates this process [4].

Frailty has been found to be a more powerful predictor of functional status in the elderly than chronological age or comorbidity, with increases in an individual's frailty greatly increasing the risk of death $[10,11]$. Frailty is also applicable in 
the diminished homeostatic reserve seen in chronic illness and organ failure. As such, frailty is an ideal characteristic for studying health outcomes in the ESLD population.

Frailty is found in many patients listed for transplantation. Lai et al. aimed to determine whether frailty predicts mortality in liver transplant candidates [4]. Four frailty assessments, including the fried frailty, short physical performance battery (SPPB), activities of daily life (ADL), and instrumental ADL (IADL) scales, were found to predict waitlist mortality in cirrhotics with at least a MELD score of 12. Although patients displaying the frail phenotype possessed statistically significantly higher MELD scores and rates of ascites and hepatic encephalopathy, there was an independent association between frailty and mortality after adjustment for liver disease severity. Frailty had only a weak positive association with MELD, suggesting that frailty is distinct from measures of severity of liver disease. High frailty scores were associated with lower survival in the absence of liver transplantation, independent of the severity of liver disease (HR 2.73, $p=$ $0.02)$. The mortality risk associated with high frailty was profound in patients with a MELD $>15$; patients with a frailty score $\geq 3$ and MELD $>15$ reached a median pre-transplant survival of 180 days.

Despite the serious implications of frailty, little research has been done to determine if frailty is a remediable condition in ESLD patients. With further study, if frailty can be shown to be remediable, targeted programs may help to improve frailty as well as to help improve quality of life in ESLD patients and potential liver transplant outcomes.

\section{Sarcopenia}

Sarcopenia, defined as a decrease in skeletal muscle mass and strength, is an important risk factor in clinical medicine associated with frailty, worse surgical and nonsurgical outcomes, and mortality [5]. It has traditionally been defined as decline associated with the aging process, but it is also of importance in that it is applicable in the diminished homeostatic reserve seen in chronic illnesses and organ failure [12]. As such, sarcopenia is an excellent metric for studying postoperative outcomes in ESLD patients.

Within recent years, sarcopenia has been observed as a particularly attractive prognostic component of frailty since it is objective and relatively easy to measure. Sarcopenia can be measured in the clinical setting using cross-sectional images to assess trunk muscle size (Fig. 1). A strong inverse correlation has been demonstrated between sarcopenia, as measured by psoas muscle area on CT imaging, and mortality following liver transplant, even after adjustment for patient demographics, size, and severity of liver disease. Total psoas area has been shown to have a significant effect on post-liver transplant mortality, with an adjusted hazard ratio (HR) of

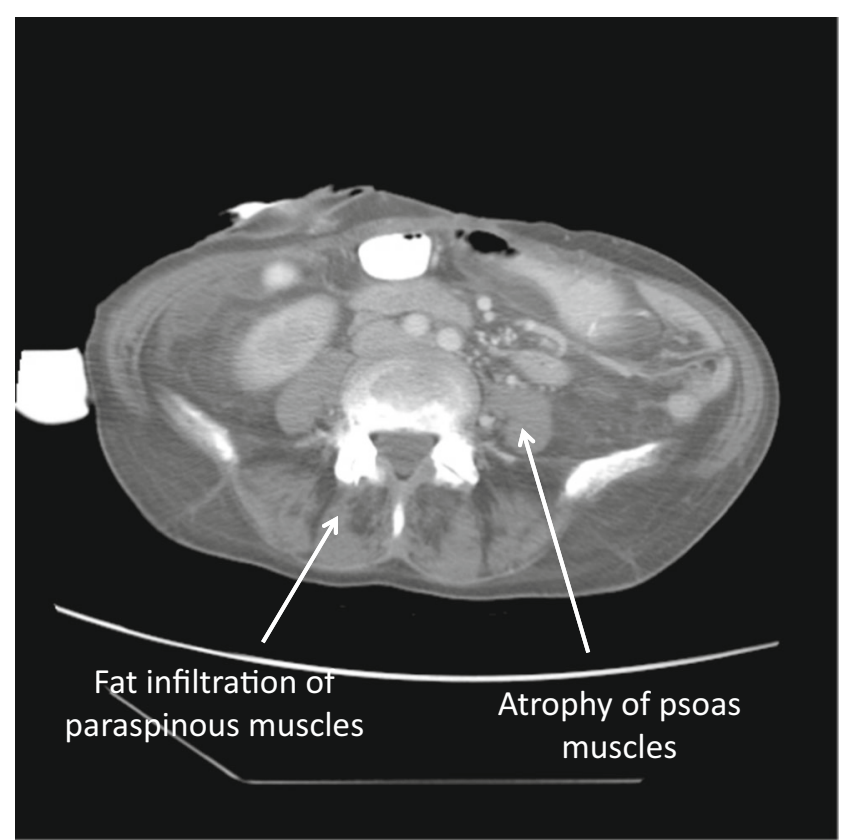

Fig. 1 Cross sectional imaging showing morphometric measurements of interest

$\mathrm{HR}=0.25[95 \%$ CI $0.12-0.47, p<0.0001]$ per $1000 \mathrm{~mm}^{2}$ increase in psoas area [5]. With all other covariates being equal among psoas area quartiles, the 1-year survival has been shown to range from $63.4 \%$ among groups with the smallest psoas area compared with $96.5 \%$ among groups with the largest psoas area. In the setting of transplantation or resection for hepatic malignancies, other studies have confirmed that sarcopenia is an independent factor predictive of postoperative complications [13, 14]. As such, sarcopenia may be particularly attractive to transplant surgeons and policy makers because its measures are objective and reproducible.

Sarcopenic patients are expensive. Decreasing trunk muscle size is independently associated with increasing payer costs $\left(\$ 6989.17\right.$ per $\left.1000 \mathrm{~mm}^{2}\right)$ [15]. Sarcopenic patients struggle to recover from complications. Among patients with complications, sarcopenic patients increased payer costs to $\$ 26,988.41$ per $1000 \mathrm{~mm}^{2}$ decrease in trunk muscle size compared to non-sarcopenic patients with complications. With respect to hospitals, negative financial margins were observed in the third of patients with the smallest trunk muscle size. Thus, sarcopenic patients represent a uniquely costly patient demographic for both payers and providers.

Despite its known impact on complications, mortality, and finances, a better understanding of sarcopenia among patients with ESLD is needed. Measures of sarcopenia could be used as a measure to influence recipient selection and help avoid futile transplants. Further, if sarcopenia could be shown to be remediable, efforts to attenuate costs and outcomes could focus on targeted preoperative interventions to optimize these high-risk patients for surgery. 


\section{Prehabilitation}

\section{Training Patients Without Liver Disease for Surgery}

The importance of a multidisciplinary surgical care pathway focusing on obtaining optimal perioperative care and outcomes has been highlighted within recent years [16]. Our institution has a surgical home program called the Michigan Surgical Home and Optimization Program (MSHOP). This program targets all potential surgical candidates.

Just as athletes train for competition, all patients need to train for surgery. Unlike numerous preoperative risk factors, which are fixed, such as medical comorbidities, a patient's physiologic state may be improved prior to major surgery. Training studies have demonstrated that even 2 weeks is considered effective, and as such, the preoperative time period is ample time for targeted intervention and physiologic optimization. MSHOP patient training targets functional status, smoking, nutrition, as well as emotional and spiritual wellness. Education and care planning are the backbone of the program and are implemented from the time of a surgical decision to the time of surgery. An interactive pedometerbased daily walking regimen with real-time data feedback with accountability through emails, text messages, or phone calls has shown promise. Achieving daily incentive spirometry is achieved through the same means, while targeted nutritional supplementation occurs through a real-time nutrition risk assessment tool. Further, the benefits of smoking cessation are heavily conveyed through education and by providing ample aid resources. As a whole, we have found these are the key factors and interventions to help patients achieve their desired results.

MSHOP has had a profound impact on preoperative optimization, and feedback from patients and families has been strongly positive. To date, over 750 patients have enrolled in the program with a $90 \%$ compliance rate. Of those patients enrolled, $85 \%$ demonstrated an increase in physical activity over the preoperative period while those who did not were already active. Smokers comprised $12 \%$ of the cohort, of which $79 \%$ were "smoke-free" at their postoperative visit due to the program. Overall, patients feel engaged with the perioperative process and incontrol of their outcomes. The financial outcomes of the program were also favorable as participating patients were matched to historical controls based on gender, ASA class, and Medicare allowable payments. Hospital costs were reduced by $\$ 2308$, while insurance payments to the hospital were reduced by $\$ 2518$, and the average length of stay was reduced by 2 days, from 6 to 4 days. As a transition is made to focusing on surgical outcomes and to bundled and capitated pay for surgical care, patient optimization and cost reduction have become increasingly important for health systems.

\section{Training Patients with ESLD Prior to Transplantation}

Current management of ESLD focuses on treating complications and tends to neglect aspects such as functional disability and frailty. This is particularly relevant in older patients with ESLD. Potentially, frailty and sarcopenia of ESLD is treatable with targeted interventions. Despite the success in training nonESLD patients prior to elective surgery described above, there is an appreciation of the complexity of training ESLD patients considering the degree of functional impairment. In a recent study, our group investigated the functional status among a prospective cohort of older individuals with cirrhosis. Among those who survived to the next interview after diagnosis, $39 \%$ had impaired ADLs, which was significantly more frequent than among controls without cirrhosis. For example, $24 \%$ of older cirrhosis patients were unable to dress and $22 \%$ were unable to bathe without assistance. Thus, it is not surprising that a significant proportion of transplant candidates appear to be unfit for high-risk surgery. Prior studies have shown that physical strength and functioning are improved after liver transplantation, but no studies have determined whether physical conditioning could be improved before transplantation - or whether this improvement would translate into better patient outcomes.

The waitlist period provides a unique opportunity to introduce key interventions that have the opportunity to optimize fitness for transplantation. Our preliminary work suggested that it is feasible to train older patients with ESLD and that the intervention should focus on walking. Our group has demonstrated that a pedometer-based walking program can be done safely, with excellent compliance, and with significant increases in daily walking distance. Among patients in the control group, we noted a decrease in energy (kcals) burned per week over the month prior to surgery and an increase in frailty scores. Conversely, patients randomized to a preoperative walking intervention maintained a stable energy utilization and had no change in frailty scores from the time of enrollment to the time of surgery. Our morphomic data suggest that a focus on exercises that utilize the thigh muscles is critical. Thigh muscle size is highly correlated with trunk muscle size $(r=0.78)$ and that walking speed is the domain within the frailty assessment is most strongly associated with mortality among cirrhotic patients.

In an attempt to build on previous work, our transplant program formulated a 10 -week pilot prehabilitation program where patients were assigned a dietician and coach and were given educational sessions regarding nutrition, protein supplements, as well as an activity-tracking device. It is important to note that nutritional interventions are likely an important component of therapy. Patients met with their coaches biweekly for a supervised exercise session and nutrition review along with additional contact either by phone or email. The program appeared feasible with measurable improvements. Patients had increased average daily activity by 2823 steps per day, greater than 1 mile $(p<0.05)$. In addition, patients were able to improve their 6-min 
walk distance by $63 \mathrm{~m}(p=0.06)$. Frailty scores were noted to have decreased or stayed stable in all patients. Of patients who were diagnosed pre-program as overweight or obese with a BMI of 27 or more, these patients lost $6 \mathrm{lb}$ on average and established improved eating habits as demonstrated by improved adherence to individualized calorie and protein goals. On an exit interview satisfaction survey, all patients reported highly favorable responses to the program (4.8/5, Likert scale), and all participants desired to continue the program. Overall, measurable improvements in physical activity and dietary habits were made in a relatively short time period. In our view, a prehabilitation program in liver transplant candidates appears feasible, with excellent adherence and patient satisfaction. Despite these favorable outcomes, further work will be required but our data suggest that efforts to improve disease management and physical conditioning could not only optimize patients for liver transplantation but could also improve outcomes among those that do not receive a transplant.

\section{Conclusion}

While liver transplantation can be life-saving for some, it may not be appropriate for high-risk patients with ESLD; the perioperative risks are high and the organ supply is limited. As frailty and sarcopenia continue to gain traction as important predictors of post-transplant morbidity and mortality, efforts to improve disease management and physical conditioning could not only optimize patients for liver transplantation but could also improve outcomes among those that do not receive a transplant. This could also result in substantial savings for payers and hospitals. Although further investigation is needed in this arena, the initial results are promising and we are confident that application of such targeted programs in older liver transplant candidates could have a profound impact on optimizing transplant outcomes.

\section{Compliance with Ethics Guidelines}

Conflict of Interest Michael Englesbe is an equity stakeholder in Prenovo LLC - a company focusing on risk stratification and perioperative optimization.

Seth Waits and Aaron Williams have no relevant conflicts.

Human and Animal Rights and Informed Consent This article does not contain any studies with human or animal subjects performed by the author.

\section{References}

1. Network OPTN. Data2015. United States Department of Health and Human Services. http://optn.transplant.hrsa.gov.

2. Freeman RB, Wiesner RH, Edwards E, et al. Results of the first year of the new liver allocation plan. Liver Transpl. 2004;10:7-15.

3. Derck JE, Thelen AE, Cron DC, et al. Quality of life in liver transplant candidates: frailty is a better indicator than severity of liver disease. Transplantation. 2015;99:340-4.

4. Lai JC, Feng S, Terrault NA, Lizaola B, Hayssen H, Covinsky K. Frailty predicts waitlist mortality in liver transplant candidates. Am J Transplant. 2014;14:1870-9.

5. Englesbe MJ, Patel SP, He K, et al. Sarcopenia and mortality after liver transplantation. J Am Coll Surg. 2010;211:271-8.

6. Waits SA, Kim EK, Terjimanian MN, et al. Morphometric age and mortality after liver transplant. JAMA Surg. 2014;149:335-40.

7. Lee CS, Cron DC, Terjimanian MN, et al. Dorsal muscle group area and surgical outcomes in liver transplantation. Clin Transplant. 2014;28:1092-8.

8. Denison HJ, Cooper C, Sayer AA, Robinson SM. Prevention and optimal management of sarcopenia: a review of combined exercise and nutrition interventions to improve muscle outcomes in older people. Clin Interv Aging. 2015;10:859-69.

9. Fried LP, Tangen CM, Walston J, et al. Frailty in older adults: evidence for a phenotype. J Gerontol A Biol Sci Med Sci. 2001;56:M146-56.

10. McAdams-DeMarco MA, Law A, King E, et al. Frailty and mortality in kidney transplant recipients. Am J Transplant. 2015;15: 149-54.

11. Arya S, Kim SI, Duwayri Y, et al. Frailty increases the risk of 30day mortality, morbidity, and failure to rescue after elective abdominal aortic aneurysm repair independent of age and comorbidities. J Vasc Surg. 2015;61:324-31.

12. Morley JE, Baumgartner RN, Roubenoff R, Mayer J, Nair KS. Sarcopenia. J Lab Clin Med. 2001;137:231-43.

13. Otsuji H, Yokoyama Y, Ebata T, et al. Preoperative sarcopenia negatively impacts postoperative outcomes following major hepatectomy with extrahepatic bile duct resection. World J Surg. 2015;39:1494-500.

14. Valero 3rd V, Amini N, Spolverato G, et al. Sarcopenia adversely impacts postoperative complications following resection or transplantation in patients with primary liver tumors. J Gastrointest Surg. 2015;19:272-81.

15. Sheetz KH, Waits SA, Terjimanian MN, et al. Cost of major surgery in the sarcopenic patient. J Am Coll Surg. 2013;217: 813-8.

16. Vetter TR, Goeddel LA, Boudreaux AM, Hunt TR, Jones KA, Pittet JF. The perioperative surgical home: how can it make the case so everyone wins? BMC Anesthesiol. 2013;13:6. 